\title{
Nelipepimut-S Plus GM-CSF Vaccine
}

National Cancer Institute

\section{Source}

National Cancer Institute. Nelipepimut-S Plus GM-CSF Vaccine. NCI Thesaurus. Code C99228.

A cancer peptide vaccine comprised of a human leukocyte antigen (HLA) A2/A3 restricted HER2/neu (ERBB2) peptide from the extracellular domain of the HER2 protein (E75 peptide) and combined with the immunoadjuvant granulocyte-macrophage colonystimulating factor (GM-CSF), with potential immunomodulating and antineoplastic activity. Upon intradermal injection, nelipepimut-S plus GM-CSF vaccine may induce a specific cytotoxic T-lymphocyte (CTL) response against HER2/neu-expressing tumor cell types. HER2/neu, a tumor-associated antigen and a member of the epidermal growth factor receptor family of tyrosine kinases, is overexpressed in various tumor cell types. GM-CSF potentiates the antitumor immune response. 